\title{
Injury to Kidney
}

National Cancer Institute

\section{Source}

National Cancer Institute. Injury to Kidney. NCI Thesaurus. Code C35245.

Trauma to the kidney. 